\title{
LÉGISLATION
}

\section{L'énergie au point de vue juridique \\ (Etat de la législation actuelle)}

Par Paul BOUGaUlT, Avocat à la Cour d'Appel de Lyon.

(SUITE)

\begin{abstract}
Après avoir exposé dans ses précédents articles la théorie de la concession simple et de la concession déclarée d'utilité publique, l'auteur consacre les lignes qui vont suivre à l'étude de la Régie directe par la commune, et il donne l'énumération des textes les plus intéressants au sujet des secours pécuniaires qui, sous forme de subventions ou de prêts, permettent aux réseaux ruraux de faire face aux lourdes charges de leur premier établissement.
\end{abstract}

\section{Exploitation en régie directe par la Commune.}

L'expression "régie directe " désigne l'exploitation d'un service par la commune elle-même, agissant par des mandataires qui sont désignés par le Maire, et courant les risques de toute entreprise; elle se trouve par là même appelée à recueillir les bénéfices, bien qu'en principe toute commune doive se préoccuper en premier lieu, d'assurer un bon service plutôt que de réaliser de gros avantages pécuniaires. Le Conseil d'Etat (voir avis des $1^{\text {er }}$ et 15 mars 1900) s'est tout d'abord prononcé contre l'exploitation, par les Municipalités, d'entreprises industrielles, pour plusieurs motifs : notamment la grande multiplicité des comptes qui rend la vérification difficile.

Mais, cette orientation s'est dans la suite modifiée (voir l'avis des Sections réunies de l'Intérieur et des Travaux Publics du 26 février 1919), et le Conseil d'Etat a été amené à dire que les communes, sous réserve de la législation spéciale en chaque matière, peuvent trouver des bénéfices dans des exploitations faites par elles-mêmes.

Par interprétation des articles 22, 24 et 26 du cahier des charges type qui permettent à la commune d'entrer, volontairement ou par suite de certaines circonstances, en possession d'un réscau, le Ministre des Travaux Publics a admis que la loi du 15 juin 1906 avait implicitement prévu qu'une commune serait amenée à gérer l'exploilation d'un réseau, et que, dès lors, il convenait de lui en donner l'autorisation. Tel est le motif pour lequel, on peut lire dans un décret du 30 août 1917, qu'il est établi un " cahier des charges " que la commune promet d'observer, étant autorisée à exploiter directęment en régie la distribution pour un usage déterminé.

Par le fait que le cahier des charges appelle cette distribution une " régie ", on est prévenu que le service qu'elle représente est nettement individualisé. Par une fiction juridique, la personne morale appelée "régie " est amenée à traiter avec la personne morale appelée "commune "; et il lui est constitué un ensemble de droits et de devoirs, par exmple, le privilège exclusif de donner l'éclairage, ce qui forcera, dans la suite, les pouvoirs publics à s'abstenir de permettre une distribution susceptible de créer une concurrence. Elle a le droil d'imposer des tarifs, qu'elle ne pourra ensuite abaisser sans étendre l'amélioration à tous les consommateurs placés dans les mèmes condịtions (puissance, horaire, mode d'utilisation). Si la régie venait à interrompre son service, le Ministre pourrait prouoncer la déchéance sans préjuArticle published by SHF and available at nttp://wWW.sht-Ihb.org dice des mesures préalables que le préfet aurait pu ordonner pour assurer la continuation du service.

Le décret réglant l'organisation de la régie signé le 8 octobre 1917, a suivi la rédaction du cahier type du 30 août 1917, et les deux documents ont paru dans le même numéro du Journal Officiel du 8 novembre 1917. Plusieurs principes peuvent se dégager de cet ensemble :

Le Préfet est le pouvoir compétent pour accorder l'autorisation de constituer en régie la distribution faite par la commune ou le Syndicat de communes dépendant du même département. Si des communes groupées dépendent de plusieurs départements, le Ministre des Travaux Publics donne l'autorisation sur avis conforme du Ministre de l'Intérieur.

Le service créé sous le nom de “ régie » est doué de la personnalité civile : il peut donc, par recettes et dépenses, établir un budget qui comprend deux sections : la première, relative au budget normal de l'exercice annuel, compte parmi ses recettes le produit brut de l'exploitation, les prélèvements faits sur un crédit qui peut ètre ouvert sur le budget ordinaire de la commune pour parer s'il y a lieu, à l'insuffisance des recettes : aux dépenses, sont inscrits les frais d'exploitation, et les charges du capital d'établissement, avec l'intérèt et l'amortissement des emprunts.

La deuxième section, relative au budget un peu anormal ou moins ordinaire que le premier, comprend : les dépenses de premier établissement, celles de travaux complémentaires et d'acquisition de matériel et porte aux recettes les sommes versées à la régie par la commune pour faire face à ces dépenses et le montant du fonds de roulement.

L'Administration de la régie comprend un Conseil de quatre membres et un Directeur nommés par le Maire avec l'agrément du Préfet, fonctions incompatibles avec tout mandat de sénateur, de député, conseiller général ou d'arrondissement, conseiller municipal conféré dans le département. Le Directeur peut être salarié ; les membres du Conseil qui se réunissent une fois par mois reçoivent une indemnité pour chaque séance à laquelle ils sont présents; les décisions du Conseil sont exécutoires dès que le Maire a fait savoir qu'il ne s'y oppose pas, et, en cas de silence de sa part, huit jours après la communication qui lui en a été fa te. Le caissier de la régie place les fonds disponibles en compte courant à la recette municipale : sa gestion est justiciable de la Cour des Comptes et assujettie aux vérifications de l'Inspecteur général des Finances. 


\section{L'Electrification dans les Campagnes. Loi du 2 Août 1923.}

Sous ce titre : "loi facilitant par des avances de l'Etat la distribution de l'énergie dans les campagnes " la loi du 2 août 1923 ( $J . O f$. du 4 août) a été votée dans un double but: créer des ressources à l'Office du Crédit National Agricole, et donner une consécration législative à la plupart des usages suivis antérieurement à sa promulgation par les Administrations compétentes : son texte suppose ces usages connus ; en effet, déjà en 1919 le Ministre de l'Agriculture et celui des Travaux Publics avaient pris l'initiative de donner des secours pécuniaires aux distributions rurales d’énergie ; cette contribution à l'établissement des réseaux, a été considérée comme une compensalion à linnovation de la loi du 16 octobre 1919 sur l'énergie hydraulique qui a supprimé aux riverains la faculté de disposer librement de leurs droils sur les eaux des rivières non classées, et par conséquent de les échanger contre une fourniture de force motrice. (Voir déclaration de M. Queuille; compte rendu de la séance de la Chąmbre des Députés du 13 mars 1923, J. Of. du 14 mars, page 1.174).

Ces secours financiers ont été réalisés par deux moyens : des prèts à un faible taux, et des subventions, soit aux communes, soit aux Sociélés agricoles quelle que soit la forme adoptée par celles-ci, la forme de la coopérative proprement dite ou celle de l'intérèt collectif agricole (circulaire du Ministre de l'Agriculture du 15 juin 1920, J. Of. du 15 juin; et du Ministre des Travaux Publics du 15 juillet 1920, J. Of. 16 juillet).

La loi du 5 août 1920 sur le crédil mutuel et la coopération agricole (Dal. 1922.4.130) ayant expressément désigné, dans son article 22, comme susceptibles d'être affiliées aux caisses de prêt agricole, toutes les Sociélés agricoles ayant pour but de doter leur région d'un réseau électrique, aucun obstacle juridique ne s'opposait à la réalisation des prêts. Mais, les demandes devinrent tellement nombreuses que beaucoup ne purent recevoir satisfaction à cause de la modicité des ressources du Crédit National Agricole; aussi, pour réglementer les conditions d'admission, le Ministre de l'Agriculture a-t-il adoplé l'usage constant de limiter le prêt à une somme égale au montant de l'emprunt que les collectivités ont su réaliser dans leur propre région; quant à la subvention qui ne peut être allouée que sur les fonds mis annuellement à la disposition du Ministre de l'Agriculture par la loi de Finances, elle a toujours été limitée à une fraction du devis total de l'établissement des ouvrages; elle ne peut être accordée qu'à la partie rurale de la distribution, d'après un coefficient variable suivant qu'il s'applique à un réseau de haule ou de basse tension, et d'après la dépense calculée par tête d'habitant desservi par ce réseau.

En ce qui concerne les prêts, l'insuffisance des ressources financières du Crédit National Agricole a été, en partie, réparée par la loi du 2 août 1923 dont les dispositions principales sont les suivantes :

$1^{\circ}$ L'Etat met à la disposition de l'Office National du Crédit Agrico'e des avances destinées à permettre à cet étab'issement public d'accorder des prêts spéciaux dont la durée ne dépasse pas quarante ans, aux communes, aux associations syndicales libres ou autorisées, aux Sociétés Coopératives, et aux Sociétés
d'Intérêt Collectif agricole qui ont pour objet l'installation ou l'exploitation des réseaux ruraux d'électricité; $2^{\circ}$ L'intérêt des prèts accordés aux collectivités sera fixé chaque année au laux du revenu domné par la rente perpétuelle comportant le laux nominal le plus élevé, d'après le cours moyen de la rente pendant. l'année précédant le prêt, sans jamais dépasser $3 \% ; 30$ leur montant ne peut, en aucun cas, excéder le capital réuni par les colleclivités emprunteuses el effectivement versé; 4o L'intérèt et l'amorlissement des prêls doivent ètre garantis par le département ou les communes.

Le total des avances pouvan ètre consenties par l'Elat à l'Office National du Crédit Agricole est fixé par la loi à 600 millions, le Ministre des Finances étant autorisé à se procurer les fonds nécessaires (dans les limiles d'un crédil ouvert charpue année par la loi de Finances) au moyen d'avances qui pourront elre faites par la Caisse de dépôts el consignations, soil sur les fonds de ses comples propres, soit sur les fonds dont elle a la gestion.

Le prêt n'exclul pas la subvention (déclaralion du Ministre de l'Agriculture à la Chambre des Députés; J.O/. 14 mars 1923 , page 1.184); celle-ci n'est donnée que sur les fonds mis a la disposilion du Ministre de IAgricullure par le Parlement el dans la mesure des disponibilités : elle est accordée d'après les coeflicients fixés à l'arrêté du Ministre de l'Agriculture du 5 janvier 1924 (J. Of. du 10 janvier) sans pouvoir, en principe, excéder $30 \%$ de la part des dépenses correspondant à des usages agricoles; loutefois, dans le cas ou les collectivités ne bénéficient pas de prêts à laux réduit, elles peuvent recevoir un complément de subvention égal à la valeur actuelle de la bonification d'annuilé correspondant au montant du prêl à laux réduit auquel elles auraient pu prétendre (art. 7 de l'arrêté ministériel).

Un décrel d'administration publique, en dale du 13 décembre 1923 ( $J$. Of. du 15 décembre) et complété par des instructions ministérielles du 5 janvier $192 . \downarrow$ (J.Of. 10 janvier) a préeisé plusieurs formalités substantielles : si les réseaux pour lesquels un prêt est sollicité, servent de transport à la distribution d'énergie électrique dont une partie seulement esl destinéc à des usages agricoles, il doit être justifié de la proportion dans laquelle cetle énergie sera employée à ces usages; el il ne peut être accordé de prêt que si cette proportion est prépondérante, l'entreprise devant couvrir par ses ressources personnelles les dépenses correspondant à une utilisation différente.

Une Commission, dont la composition est déterminée par l'art. 9 est instituée pour examiner les avant-projets et les projets des travaux qui ont été, après instruction et les avis concordants du contrôle des distributions d'énergie électrique el du service du génie rural, pris en considération par le service de l'agriculture; quand il existe un désaccord entre les deux services ci-dessus visés, la prise en considération ne peut intervenir qu'après entenle entre le Ministre des. Travaux Publics el lo Ministre de l'Agriculture.

La loi du 2 août 1923 ayant décidé que le maximum du prêt ne peut dépasser le montant des emprunts réalisés par la collectivité intéressée, le décret fixe en deça de ce maximum le pourcentage du prêt; il ne sera égal au maximum que si la dépense de caractère agricole atteint une limite annuellement fixée par le Ministre de l'Agriculture el le Ministre des Travaux Publics. 